\title{
Interação alelopática de Jatropha curcas L. com Helianthus annuus L. e Zea mays L. por meio de exsudados radiculares
}

\author{
Paulo Sérgio Siberti da Silva ${ }^{1 *}$ \\ Andréa Maria Teixeira Fortes ${ }^{2}$ \\ Nayara Parizoto Boiago ${ }^{3}$ \\ Daiane Maria Pillati ${ }^{3}$ \\ Fernanda Melo Gomes ${ }^{4}$

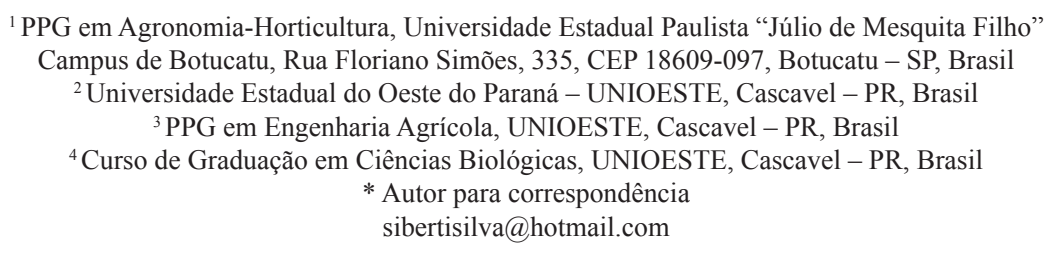

Submetido em 22/10/2011

Aceito para publicação em 16/04/2012

\section{Resumo}

O objetivo deste trabalho foi avaliar o efeito da interação alelopática de Jatropha curcas com Helianthus annuus e Zea mays via exsudados radiculares bem como seus efeitos nas sementes das espécies. Foram utilizadas quatro repetições, como substratos para germinação das sementes foram utilizados papel germiteste previamente autoclavado e antes da montagem dos experimentos foi realizada quebra de dormência das sementes de $H$. annuus via pré-resfriamento em congelador por sete dias a $5^{\circ} \mathrm{C}$. As sementes de $J$. curcas foram colocadas para germinarem por oito dias em câmara de germinação em condições de temperatura e fotoperíodo controlados. Posteriormente, as plântulas foram descartadas e no mesmo substrato adicionadas, por repetição, 50 sementes de H. annuus, utilizando como testemunha apenas água destilada. Nos experimentos dos exsudados radiculares de H. annuus sobre $J$. curcas e da interação alelopática entre $J$. curcas e Z. mays foi realizada a mesma metodologia. Após a análise estatística, pode-se verificar que $J$. curcas apresenta efeito inibidor sobre o tempo e velocidade média de germinação das plântulas de H. annuus, já Z. mays apresenta efeito inibidor sobre a germinação das sementes de J. curcas.

Palavras-chave: Agricultura familiar; Alelopatia; Consórcio

\section{Abstract}

Allelopathic interaction of Jatropha curcas $\mathbf{L}$. with Helianthus annuus $\mathbf{L}$. and Zea mays $\mathbf{L}$. The goals of this work were to evaluate the effect of the allelopathic interaction of Jatropha curcas with Helianthus annuus and Zea mays from root exudates, as well as to evaluate how these exudates influenced the seeds of these species. Four replicates were made with seeds that were germinated in germiteste paper, which was previously autoclaved. Dormancy of the $H$. annuи seeds was broken by pre-cooling them in a freezer for seven days at $5^{\circ} \mathrm{C}$. 
Jatropha curcas seeds were cultivated for eight days in a germination chamber under a controlled temperature and photoperiod. These seedlings were then discarded and the same substrate was used to germinate $50 \mathrm{H}$. annuus seeds; only distilled water was used as the control. The root exudates of $H$. annuus tested on J. curcas and the allelopathic interaction between $J$. curcas and Z. mays were carried out using the same methodology. Statistical analysis indicated that $J$. curcas has an inhibitory effect on the time and average speed of germination of $H$. annuus seedlings and $Z$. mays has an inhibitory effect on the germination of the seeds of $J$. curcas.

Key words: Allelopathy; Family farming; Intercrop

\section{Introdução}

Jatropha curcas L., pertencente à família Euphorbiaceae, é considerada uma oleaginosa promissora para fabricação de biocombustível, popularmente conhecida como pinhão-manso, a espécie apresenta sementes ricas em óleo (MAKKAR; BECKER, 2009) e embora ainda não tenha seu zoneamento agrícola definido, é considerada pelos pequenos produtores uma planta atrativa para inserção na cadeia produtora familiar (CASTRO et al., 2008).

Foi introduzida em alguns países tropicais e cresce naturalmente em países da América equatorial, podendo ser consorciada com espécies cultivadas, quando em particular com amendoim (Arachis hypogaea L.), pimenta-verde (Piper nigrum L.), gergelim (Sesamum indicum L.) e girassol (Helianthus annuus L.) (REJILA; VIJAYAKUMAR, 2011). Além destas espécies, outra opção de consórcio com a planta é Zea mays L., o milho. Esta gramínea corresponde a $37 \%$ da produção nacional de grãos, sendo também insumo básico na avicultura e suinocultura (PINAZA, 2007). Devido esta versatilidade de uso é muito utilizado em sistemas de agricultura familiar, se tratando de um dos mais importantes produtos do setor agrícola brasileiro (CRUZ et al., 2006).

Outra oleaginosa também fonte de biodiesel, $H$. annuus é uma Asteraceae comumente utilizada para produção de óleo comestível. É uma planta que, devido estas características, ganha cada vez mais espaço nas pequenas propriedades vinculadas ao método familiar de cultivo, sendo muito indicada pelos produtores como uma cultura alternativa em consórcios complementando as culturas já estabelecidas, além do fato que, da massa resultante da extração de seu óleo, faz-se uma torta de valor altamente proteico usada para alimentação animal (SAIZ, 2009).
Ao realizar o plantio intercalado de $J$. curcas com H. annuus, Castro et al. (2008) verificaram que houve diminuição do diâmetro dos capítulos e peso de mil aquênios do cultivar de $H$. annuus estudado, obtendo 19 cm de diâmetro de capítulo e peso de mil aquênios de 67g. Segundo a CATI - Coordenadoria de Assistência Técnica Integral (2011), em condições ótimas o cultivar utilizado pode produzir capítulos de $22 \mathrm{~cm}$ e peso de mil aquênios de $70 \mathrm{~g}$.

Por ser perene, assim como as demais espécies com esta característica, J. curcas fica exposta por longos períodos às vicissitudes do ambiente como patógenos $\mathrm{e}$ predadores, levando no decorrer do tempo que a planta desenvolvesse metabólitos secundários que a protegesse da maioria destes ataques, onde, no ecossistema, tais moléculas podem exercer nas plantas circundantes o fenômeno da alelopatia (FERREIRA; ÁQUILA, 2000).

Investigações sobre os fitoquímicos de extratos da casca do caule da planta realizadas por Igbinosa et al. (2009) mostraram presença de alguns metabólitos secundários como esteróides, taninos, glicosídeos, alcalóides e flavonóides. Já Begum et al. (2011) identificaram $\beta$-sitosterol-3-O- $\beta$-Dglucopyranoside (daucosterin), 7-keto- $\beta$-sitosterol e $\beta$-sitosterol através de análises em extrato foliar. Para esta espécie, foi constatado que os compostos fenólicos são as principais substâncias responsáveis por exercerem efeito alelopático, onde em altas concentrações de seu extrato foliar e radicular foi verificado a inibição do crescimento das plântulas de feijão (Phaseolus vulgaris L.), milho (Zea mays L.), tomate (Solanum lycopersicum L.) e quiabo (Abelmoschus esculentus (L.) Moench.) (ABUGRE; SAM, 2010).

Praticado ao longo dos anos, o consórcio não é somente uma prática realizada por razões tradicionais, mas, também, por certas vantagens que coadjuvaram 
na sua adaptação ecológica, se tratando de um método comum entre agricultores de regiões tropicais do mundo, onde, se planta simultaneamente numa mesma área, duas ou mais espécies cultivadas (BEZERRA et al., 2007).

Levando em conta o ponto de vista agronômico, um consorciamento entre culturas básicas e espécies oleaginosas pode promover maior produtividade e renda, pois as culturas básicas lucram da nutrição e efeito abrigo quando em consórcio com espécies perenes (MAKKAR; BECKER, 2009). No entanto, para que o método seja bem aproveitado, as culturas devem estar bem relacionadas entre si, o plantio e desenvolvimento de uma espécie não devem interferir negativamente em outra (PINTO et al., 2011). Assim, a alelopatia se trata de uma importante ferramenta para a verificação de afinidades ou prejuízos num sistema de plantio, estudando as relações entre as plantas e estas com os micro-organismos (FERREIRA; ÁQUILA 2000).

O termo foi cunhado por Hans Molisch em 1937, é definido como a influência benéfica ou maléfica, de um indivíduo sobre outro, onde o efeito é mediado por biomoléculas denominadas aleloquímicos. Estes compostos são oriundos do metabolismo secundário vegetal e liberados no ambiente por lixiviação, volatilização ou exsudados pelas raízes das plantas (RIZVI; RIZVI, 1992). Quando liberados, podem influenciar no crescimento e desenvolvimento de sistemas biológicos circundantes (RAZAVI, 2011) e, na agricultura, podem até mesmo ser utilizados no controle de plantas indesejáveis (APPLETON; BERRIER, 2009), visando obter um melhor rendimento de produção.

Culturas que têm atividade alelopática podem ser utilizadas em sistemas de rotação, como H. annuus, que quando cultivado antes da soja (Glycine max (L.) Merr.), reduz a quantidade de espécies infestantes (PASQUALETO et al., 2007). Para esta espécie, dentre as substâncias com atividade alelopática comprovada estão os terpenos e fenóis (CORSATO et al., 2010), substâncias que apresentam funções importantes, como combate a herbívoros, insetos e fungos, além de influenciar no crescimento de outros vegetais (TAIZ; ZEIGER, 2009).
Trabalhos também relatam o potencial alelopático de Z. mays, onde extratos aquosos na concentração de $5 \%(\mathrm{p} / \mathrm{v})$ reduziram o índice de velocidade de germinação, comprimento de radícula e hipocótilo de plântulas de alface (Lactuca sativa L.) ao passo que, em casa de vegetação, a palha do híbrido GNZ 2004 afetou significativamente o teor de clorofila e a biomassa seca do caule de café (Coffea arabica L.), cultivar Catuaí (FRANÇA, 2007).

Para avaliação do potencial alelopático de uma determinada espécie usam-se como procedimentos os bioensaios, onde parâmetros globais como germinação, crescimento e desenvolvimento das plântulas ou plantas adultas e parâmetros mais específicos, como atividade de alguns processos fisiológicos, por exemplo, fotossíntese, respiração e conteúdo de clorofila podem ser avaliados (SOUZA-FILHO; ALVES 2002).

Na germinação os efeitos dos aleloquímicos estão associados ao estresse oxidativo, devido formação de espécies reativas de oxigênio que atuam de forma direta ou como sinalizadores nos processos de degradação celular, gerando efeitos danosos a processos fisiológicos vitais, refletindo em alterações no desenvolvimento inicial das plântulas, estádio fundamental para obtenção de uma uniformidade da cultura (ALMEIDA et al., 2008).

O potencial de ação destas moléculas está diretamente relacionado com o seu grupo funcional e suas propriedades químicas (BARBOSA et al., 2005). Alguns compostos somente atuam quando em presença de outros, portanto, são específicos em combinação e proporção, ou seja, atuam em sinergismo, pois apenas um composto secundário não atinge a concentração mínima necessária para exercer efeito na planta receptora (ALMEIDA, 1988).

Desta forma, testes que agregam procedimentos experimentais que caracterizem a propriedade alelopática de determinadas culturas servem para contribuir para estudos de dinâmicas entre espécies e elaboração de estratégias de manejo e produção (SOUZA-FILHO et al., 2010). Além disso, possibilita ao pesquisador identificar possíveis insucessos no desenvolvimento de determinadas culturas em sistemas agroecológicos e 
contribui para adoção de espécies que reduzem custos de manejo, promovendo diminuição do uso de defensivos químicos evitando os impactos que os mesmos causam no ecossistema (GOLDFARB et al., 2009).

Assim, em função do exposto, o objetivo do trabalho é avaliar, em laboratório, o efeito, da interação alelopática de $J$. curcas com $H$. annuus e Z. mays , oriundo de compostos liberados via exsudados radiculares de suas plântulas, bem como os seus efeitos nas sementes das espécies.

\section{Material e Métodos}

Os experimentos foram desenvolvidos no Laboratório de Fisiologia e Ecologia Vegetal da Universidade Estadual do Oeste do Paraná - UNIOESTE, campus de Cascavel-PR a partir de agosto de 2008. As sementes de $J$. curcas foram doadas pela Empresa Rural Biodiesel de Eldourado, MS, as de H. annuus (cultivar IAC-IARAMA) pela empresa Perá Sementes de Piracicaba, SP, e as de Z. mays (cultivar FTH 950) pela empresa Coopavel Cooperativa Agroindustrial de Cascavel, PR.

Antes da instalação do experimento, os papéis para germinação foram previamente autoclavados a temperatura de $121^{\circ} \mathrm{C} \pm 2^{\circ} \mathrm{C}$ por $20 \mathrm{~min}$ e em seguida, acondicionados em estufa de secagem com temperatura entre $50^{\circ} \mathrm{C} \pm 3^{\circ} \mathrm{C}$. Posteriormente os papéis foram pesados em balança de precisão e o seu peso multiplicado por 2,5 para determinar a quantidade de água necessária para umedecê-los (BRASIL, 2009).

Foi realizada a quebra de dormência das sementes de $H$. annuus através de pré-resfriamento em congelador por sete dias a $5^{\circ} \mathrm{C}$ e as sementes de $J$. curcas escarificadas manualmente do lado oposto da micrópila. Foram utilizados dois tratamentos, cada um com quatro repetições, o tratamento contendo exsudado e o controle, onde foi adicionada apenas água destilada. Como padrão, para cada repetição de cada tratamento, foram usadas 50 sementes de H. annuus e Z. mays (BRASIL, 2009) e 15 sementes de J.curcas, totalizando por tratamento, 200 sementes de H. annuus e Z. mays e 60 sementes de J.curcas.
No experimento onde se avaliou o efeito alelopático do exsudado radicular de J.curcas sobre $H$. annuus e Z. mays, para a obtenção do exsudado radicular de J.curcas, 15 sementes da espécie, por repetição, durante oito dias, foram colocadas para germinarem e após este período, suas plântulas foram descartadas e nos mesmos substratos adicionadas para germinarem, de acordo com as Regras para Análises de Sementes (BRASIL, 2009), durante sete dias, as sementes de $H$. annuus e $J$. curcas para os estudos de alelopatia.

Na segunda etapa, no experimento onde se avaliou o efeito alelopático dos exsudados radiculares de $H$. annuus e Z. mays sobre J. curcas, para a obtenção dos exsudados radiculares de H. annuus e Z. mays, 50 sementes, por repetição, de cada espécie foram colocadas para germinarem durante sete dias (BRASIL, 2009), sendo, após este período, suas plântulas descartadas e adicionadas nos mesmos substratos as sementes de $J$. curcas por 10 dias para o estudo de alelopatia.

Os experimentos foram acondicionados em câmara de germinação com fotoperíodo de $12 \mathrm{~h}$ de claro e $12 \mathrm{~h}$ de escuro com temperatura controlada a $25^{\circ} \mathrm{C} \pm 3^{\circ} \mathrm{C}$. Foram realizadas avaliações diárias iniciando as avaliações 24h após a semeadura, sendo considerada germinada a semente que apresentasse no mínimo $2 \mathrm{~mm}$ de radícula (HADAS, 1976).

O delineamento experimental adotado foi inteiramente casualisado e após a realização dos experimentos foram feitos cálculos dos parâmetros porcentagem de germinação (PG), tempo médio de germinação (TMG), velocidade média de germinação (VMG) e comprimento da maior raiz (CMR), sendo este último parâmetro avaliado somente para as plântulas de $H$. annuus e $Z$. mays quando submetidas ao exsudado de raiz de $J$. curcas, pois como a última se trata de uma espécie arbórea, algumas de suas sementes demoram muito para germinar e acabam deteriorando, logo, sementes deterioradas foram descartadas para não prejudicar o restante das sementes, medindo as raízes somente das espécies cultivadas.

Calculou-se o tempo médio de germinação (TMG) segundo Edmond e Drapalla (1958) e a velocidade média de germinação (VMG) segundo Laboriau (1983). Em 
sequência aos cálculos dos parâmetros, todos os dados obtidos foram submetidos à análise de variância (Teste F) e para a variável porcentagem de germinação (PG) foi realizada a transformação do arco seno da raiz quadrada de $\mathrm{x} / 100$ (PIMENTEL-GOMES, 1990) e as médias comparadas pelo teste de Tukey a $5 \%$ de probabilidade.

\section{Resultados e Discussão}

Observando os dados na Tabela 1 é possível verificar no parâmetro porcentagem de germinação que mesmo não diferindo significativamente, o exsudado de raiz de $J$. curcas proporcionou uma diminuição da germinação das sementes de $H$. annuus, havendo diferenças significativas para o tempo e velocidade média de germinação, indicando atraso no crescimento das plântulas. Isto se torna visível nos dados referentes ao comprimento da maior raiz, onde mesmo não diferindo, foram observadas raízes menores no tratamento contendo exsudado.

TABELA 1: Porcentagem de germinação (PG), tempo médio de germinação (TMG) e velocidade média de germinação (VMG) de sementes de Helianthus annuus L. submetidas a exsudado de raiz de Jatropha curcas L. Cascavel - PR.

\begin{tabular}{ccccc}
\hline Tratamentos & PG (\%) & $\begin{array}{c}\text { TMG } \\
\text { (Dias) }\end{array}$ & $\begin{array}{c}\text { VMG } \\
\text { (Sementes/ } / \\
\text { Dias) }\end{array}$ & $\begin{array}{c}\text { CMR } \\
\text { (cm) }\end{array}$ \\
\hline Testemunha & $88 \mathrm{a}$ & $1,74 \mathrm{~b}$ & $0,57 \mathrm{a}$ & $8,05 \mathrm{a}$ \\
Exsudado & $81 \mathrm{a}$ & $2,34 \mathrm{a}$ & $0,43 \mathrm{~b}$ & $5,95 \mathrm{a}$ \\
\hline C.V(\%) & 10,74 & 12,27 & 9,46 & 40,56 \\
\hline
\end{tabular}

Médias seguidas da mesma letra não diferem significativamente entre si pelo teste Tukey, ao nível de $5 \%$ de probabilidade. C.V (\%) = coeficiente de variação.

De acordo com Ferreira (2004), a porcentagem final da germinação é, normalmente, a menos influenciada quando em presença dos aleloquímicos, no entanto, o tempo médio de germinação e a velocidade média de germinação são mais sensíveis, onde muitas vezes ocorrem alterações na curva de germinação.

Isso foi verificado no atual trabalho, onde o tempo médio de germinação e a velocidade média de germinação das plântulas de $H$. annuus foram os estádios onde realmente o exsudado de raiz de $J$. curcas exerceu interferência alelopático (Tabela 1).

O efeito alelopático de $J$. curcas no desenvolvimento inicial também foi verificado por Rejila e Vijayakumar (2011) em experimentos com extrato aquoso de folhas da planta sobre o crescimento de pimentão (Capsicum annum L.), havendo efeito inibitório sobre o crescimento das plântulas, ao passo que, com o aumento das concentrações, menores foram os tamanhos das plântulas de pimentão, obtendo valores de parte aérea entre $0,1 \mathrm{~cm}$ a $1,7 \mathrm{~cm}$ para o extrato a $20 \%$.

Outros trabalhos comprovam a alelopatia de J. curcas em representantes de Asteraceae, como o realizado por Wang et al. (2009), que relatam o efeito alelopático dos lixiviados das folhas de pinhão-manso no desenvolvimento de plântulas de Tagetes erecta L., conhecida popularmente como cravo-amarelo. Os lixiviados, segundo os autores, proporcionaram diminuição do comprimento da parte aérea e raízes das plântulas avaliadas e quando foram adicionados resíduos de $J$. curcas no solo, houve um aumento da toxicidade do mesmo, exercendo maior fitotoxidade no sistema radicular das plântulas. Outro exemplo é o estudo realizado por Lemos et al. (2009), que verificaram mesmo em pequenas concentrações, efeito alelopático de extrato das folhas de $J$. curcas na germinação de sementes de alface.

Estudando o efeito alelopático de extratos de folhas e raizes de algumas espécies vegetais, Abugre et al. (2011) concluíram que $J$. curcas proporcionou reduções significativas da extensão da plúmula e radícula dos cultivares de Z. mays, tomate (Solanum lycopersicum L.) e feijão-de-corda (Vigna unguiculata (L.) Walp) utilizados. Ressaltando também que, de todas as espécies avaliadas no trabalho, foi a espécie que mais inibiu a germinação das sementes de quiabo (Abelmoschus esculentus (L.) Moench), tendo o extrato de suas raízes inibido $53,3 \%$ do lote de sementes e o extrato foliar $68,3 \%$, o que evidenciou, um efeito mais acentuado do extrato foliar quando comparado ao do extrato radicular.

Estes dados corroboram com os resultados obtidos no atual trabalho, onde foi verificado o efeito alelopático de $J$. curcas. No entanto, ainda não se encontram 
trabalhos relacionados ao efeito alelopático do exsudado radicular da espécie em literatura.

Quanto aos resultados obtidos para o experimento de exsudado de raiz de $H$. annuus sobre $J$. curcas foi constatado que o exsudado de $H$. annuus não interferiu significativamente no percentual de germinação, tempo médio de germinação e velocidade média de germinação das plântulas de $J$. curcas (Tabela 2).

TABELA 2: Porcentagem de germinação (PG), tempo médio de germinação (TMG) e velocidade média de germinação (VMG) de plântulas de Jatropha curcas L. submetidas a exsudado de raiz de Helianthus annuus L. Cascavel - PR.

\begin{tabular}{cccc}
\hline Tratamentos & PG(\%) & TMG(Dia) & $\begin{array}{c}\text { VMG } \\
\text { (Sementes/ } \\
\text { Dias) }\end{array}$ \\
\hline Testemunha & $75 \mathrm{a}$ & $2,97 \mathrm{a}$ & $0,33 \mathrm{a}$ \\
Exsudado & $86 \mathrm{a}$ & $3,28 \mathrm{a}$ & $0,31 \mathrm{a}$ \\
\hline CV $(\%)$ & 9,54 & 11,12 & 12,56 \\
\hline
\end{tabular}

Médias seguidas da mesma letra não diferem significativamente entre si pelo teste Tukey, ao nível de 5\% de probabilidade. CV (\%) = coeficiente de variação.

No entanto, é constatado em bibliografia que a planta apresenta propriedades alelopáticas (ZENG et al., 2008; NASEEM et al., 2009), como exemplo Gavazza et al. (2008), que observaram um estímulo no desenvolvimento e crescimento das raízes adventícias secundárias de Z. mays quando as plântulas foram submetidas ao extrato das folhas de $H$. annuus em concentrações de 1,$5 ; 3$ e $6 \%$, respectivamente.

Silva et al. (2009) também verificaram o efeito alelopático da espécie no crescimento de picãopreto (Bidens pilosa L.), corda-de-viola (Ipomoea grandifolia (Dammer) O’Donel.), trigo (Triticum aestivum L., cultivar BRS 208) e S. lycopersicum L., porém, não constataram diferenças significativas entre as porcentagens de germinação de quaisquer das espécies avaliadas quando semeadas no mesmo substrato que $H$. annuus via método de substituição, o que foi verificado no atual trabalho e confirma novamente a afirmação de Ferreira (2004), de que a porcentagem de germinação é o parâmetro menos influenciado pelos aleloquímicos.
Também não foi constatado efeito alelopático do exsudado de raiz de $J$. curcas na germinação e crescimento inicial de Z. mays (Tabela 3). No levantamento bibliográfico, não foram encontrados trabalhos relacionados à interação alelopática ou cultivo em consórcio entre estas espécies estudadas.

TABELA 3: Porcentagem de germinação (PG), tempo médio de germinação (TMG) e velocidade média de germinação (VMG) de sementes de Zea mays L. submetidas a exsudado de raiz de Jatropha curcas L. Cascavel - PR.

\begin{tabular}{ccccc}
\hline Tratamentos & PG (\%) & $\begin{array}{c}\text { TMG } \\
\text { (Dias) }\end{array}$ & $\begin{array}{c}\text { VMG } \\
\text { (Sementes/ } \\
\text { Dias) }\end{array}$ & $\begin{array}{c}\text { CMR } \\
\text { (cm) }\end{array}$ \\
\hline Testemunha & $95,5 \mathrm{a}$ & $1,97 \mathrm{a}$ & $0,51 \mathrm{a}$ & $6,84 \mathrm{a}$ \\
Exsudado & $98,0 \mathrm{a}$ & $1,78 \mathrm{a}$ & $0,60 \mathrm{a}$ & $10,39 \mathrm{a}$ \\
\hline C.V(\%) & 5,22 & 17,87 & 27,32 & 28,48 \\
\hline
\end{tabular}

Médias seguidas da mesma letra não diferem significativamente entre si pelo teste Tukey, ao nível de $5 \%$ de probabilidade. C.V (\%) = coeficiente de variação.

Já quando as sementes $J$. curcas foram submetidas ao exsudado de raiz de $Z$. mays houve efeito alelopático, havendo diferença significativa para a porcentagem de germinação quando em presença do exsudado, onde menos da metade das sementes de $J$. curcas semeadas germinaram (Tabela 4).

TABELA 4: Porcentagem de germinação (PG) tempo médio de germinação (TMG) e velocidade média de germinação (VMG) de sementes de Jatropha curcas L. submetidas a exsudado de raiz de Zea mays L. Cascavel - PR.

\begin{tabular}{cccc}
\hline Tratamentos & PG (\%) & TMG (Dias) & $\begin{array}{c}\text { VMG } \\
\text { (Sementes/ } \\
\text { Dias) }\end{array}$ \\
\hline Testemunha & $75 \mathrm{a}$ & $2,97 \mathrm{a}$ & $0,33 \mathrm{a}$ \\
Exsudado & $46 \mathrm{~b}$ & $3,40 \mathrm{a}$ & $0,29 \mathrm{a}$ \\
\hline C.V(\%) & 11,46 & 9,57 & 10,69 \\
\hline
\end{tabular}

Médias seguidas da mesma letra não diferem significativamente entre si pelo teste Tukey, ao nível de 5\% de probabilidade. C.V (\%) $=$ coeficiente de variação.

Esta inibição da germinação foi causada, provavelmente, pela liberação de aleloquímicos 
exsudados pelas plântulas de Z. mays, causando alterações neste processo.

Estes resultados concordam com os dados obtidos por Santos et al. (2003), que em condições de casa de vegetação verificaram o efeito alelopático de Z. mays, onde a palhada de três genótipos (cultivares AG1051, C333 e C435) quando incorporadas no solo inibiram a área foliar, altura de planta e diâmetro de caule das plantas de cafeeiro (Coffea arabica L.) até 60 dias após o plantio (DAP), ao passo que os valores de biomassa seca das raízes foram menores na presença da palhada do cultivar AG1051 aos 180 DAP.

Logo, em um agroecossistema esta interação entre plantas pode influenciar direta ou indiretamente na produtividade e permanência sadia das culturas (PIÑA-RODRIGUES; LOPES 2001) e para que seja comprovado que as espécies estudadas têm efeito alelopático, sugerem-se estudos a campo para verificar a intensidade desse efeito.

Nas condições em que foram conduzidos os experimentos, conclui-se que em laboratório, $J$. curcas apresenta efeito inibidor sobre o tempo e velocidade média de germinação de H. annuus, já Z. mays apresenta efeito inibidor sobre a germinação das sementes de $J$. curcas.

\section{Referências}

ABUGRE, S.; APETORGBOR, A. K.; ANTWIWAA, A.; APETORGBOR, M. M. Allelopathic effects of ten tree species on germination and growth of four traditional food crops in Ghana. Journal of Agricultural Technology, Tehran, v. 7, n. 3, p. 825834, 2011.

ABUGRE, S.; SAM, S. J. Q. Evaluating the allelopathic effect of Jatropha curcas aqueous extract on germination, radicle and plumule length of crops. International Journal of Agriculture and Biology, Faisalabad, v. 12, n. 5, p. 769-772, 2010.

ALMEIDA, F. S. A. Alelopatia e as plantas. Londrina: IAPAR, 1988. 60 p. (Circular, n. 53).

ALMEIDA, G. D. de; ZUCOlOTO, M.; ZETUN, M. C.; COELHO, I.; SOBREIR, F. M. Estresse oxidativo em células vegetais mediante aleloquímicos. Revista Facultad Nacional de Agronomia, Medellín, v. 61, n. 1, p. 4237-4247, 2008.

APPLETON, B.; BERRIER, R. The walnut tree: Allelopathic effects and tolerant plants. Virginia: College Of Agriculture And Life Sciences, Virginia Polytechnic Institute And State University, 2009. Disponível em < http://pubs.ext.vt.edu/430/430-021/430-021_ pdf.pdf $>$. Acesso em: 23 junho 2011.
BARbosa, L. C. A.; MAlthA, C. R. A.; DEMUNER, A. J.; GANEM, F. R. Síntese de novas fitotoxinas derivadas do 8-oxabiciclo[3.2.1]oct-6-en-3-ona. Química Nova, São Paulo, v. 28, n. 3, p. 444-450, 2005.

BEGUM, M.; SOHRAB, M. H.; AFROZ, F.; AKTER, S.; HASAM, C. M.; CHOWDHURY A. M. S. Phytochemical investigations on the leaves of Jatropha curcas. Dhaka University Journal of Science, Dhaka, v. 59, n. 1, p. 61-63, 2011.

BEZERRA, A. P. A.; PITOMBEIRA, J. B.; TÁVORA, F. J. A. F.; VIDAL NETO, F. C. Rendimento, componentes da produção e uso eficiente da terra nos consórcios sorgo $\mathrm{x}$ feijão-de-corda e sorgo $\mathrm{x}$ milho. Revista Ciência Agronômica, v. 38, n. 1, p. 104-108, 2007.

BRASIL. Ministério da Agricultura, Pecuária e Abastecimento. Regras para análise de sementes. Brasília: Secretaria de Defesa Agropecuária, 2009. 399 p.

CASTRO, C. M.; DEVIDE, A. C. P.; ANACLETO, A. H. Avaliação de acessos de pinhão manso em sistema de agricultura familiar. Revista Tecnologia \& Inovação Agropecuária, São Paulo, v. 1. n. 2, p. 41-49, 2008. Disponível em <http://www.dge.apta.sp.gov. br/publicacoes/T\&IA2/T\&IAv1n2/Artigo_Pinh\%C3\%A3o_ Manso_4.pdf>. Acesso em: 13/09/2011.

CATI - Coordenadoria de Assistência Técnica Integral. Centro de Produção de Sementes. Departamento de Sementes, Mudas e Matrizes/CATI (Campinas, SP). Girassol Multissol. 2011. Disponível em <http://www.cati.sp.gov.br/Cati/_produtos/ SementesMudas/cultivares/GIRASSOL-MULTISSOL.pdf>. Acesso em: 24 junho 2011.

CORSATO, J. M.; FORTES, A. M. T.; SANTORUM, M.; LESZCZYNSKI, R. Efeito alelopático do extrato aquoso de folhas de girassol sobre a germinação de soja e picão-preto. Semina: Ciências Agrárias, Londrina, v. 31, n. 2, p. 353-360, 2010.

CRUZ, J. C.; KONZEN, E. A.; FILHO, I. A. P.; MARRIEL, I. E. Produção de milho orgânico na agricultura familiar. Sete Lagoas: Embrapa Milho e Sorgo.17 p. 2006. (Circular, n. 81).

EDMNOND, J. B.; DRAPALLA, W. J. The effects of temperature, sana and soil, and acetone on germination oj okra seed. Proceedings of the American Society for Horticultural Science, Alexandria, v. 71, p. 428-443, 1958.

FERREIRA, A. G. Interferência: Competição e alelopatia. In: FERREIRA, A. G.; BORGHETTI, F. Germinação do básico ao aplicado. Porto Alegre: Artmed, 2004. p. 251-262.

FERREIRA, A. G.; AQUILA, M. E. A. Alelopatia: Área emergente da ecofisiologia. Revista Brasileira de Fisiologia, Rio de Janeiro, v. 12, p. 175-204, 2000.

FRANÇA, A. C. Potencial alelopático de híbridos de milho no desenvolvimento inicial de caffeiros (Coffea arábica L.). 2007. 69 f. Dissertação (Mestrado em Agronomia) - Universidade Federal de Lavras, Lavras. 2007.

GAVAZZA, M. I. A.; BRITO, R.; ROSSETTO, C. A. V. Germinação e vigor de sementes submetidas aos extratos aquosos de folhas de girassol. Revista de Ciências da Vida, Rio de Janeiro, v. 28, n. 2, p. 11-18, 2008.

GOLFARD, M.; PIMENTEL, L. W.; PIMENTEL, N. W. Alelopatia: Relações nos agrossistemas. Revista Tecnologia \& Ciência Agropecuária, João Pessoa, v. 3, n. 1, p. 23-28, 2009.

HADAS, A. Water uptake and germination of leguminous seeds under chaning external water potential in osmotic solution. Journal 
Experimental of Botany, Oxford, v. 27, p. 480-489, 1976.

IGBNOSA, O. O.; IGNINOSA, E. O.; AIYEGORO, O. A. Antimicrobial activity and phytochemical screening of stem bark extracts from Jatropha curcas (Linn). African Journal of Pharmacy and Pharmacology, Nairobi, v. 3, n. 2, p. 58-62, 2009.

LABORIAU, L. G. A Germinação das sementes. Washington: Organização dos Estados Americanos, 1983. 174 p. (Monografia, 24).

LEMOS, J. M.; MEINERZ, C. C.; BERTUOL, P.; CORTEZE, O.; GUIMARÃES, V. F. Efeito alelopático do extrato aquoso de folha de pinhão manso (Jatropha curcas L.) sobre a germinação e desenvolvimento inicial de alface (Lactuca sativa cv. Grand Rapids). Revista Brasileira de Agroecologia, Porto Alegre, v. 9, n. 2, p. 2529-2523, 2009.

MAKKAR, H. P. S.; BECKER, K. Jatropha curcas, a promising crop for the generation of biodiesel and value-added coproducts. European Journal of Lipid Science and Technology, Weinheim, v. 111, p. 773-787, 2009.

NASEEM, M.; ASLAM, M.; ANSAR, M.; AZHAR, M. Allelopathic effects of sunflower water extract on weed control and wheat productivity. Pakistan Journal of Weed Science Research, Peshawar, v. 15, n. 1, p. 107-116, 2009.

PASQUALETO, A.; COSTA, A. L. M.; SILVA, A. A.; SIDIYMA, C. S. Ocorrência de plantas daninhas da cultura de soja (Glycine max (L.) Menrril) em sucessão as culturas de safrinha no sistema de plantio direto. 2007. Disponível em $<$ http://agata. ucg;br/formulários/ucg/docentes/eng/pasqualeto/artigos_48.pdf>. Acesso em: 12 setembro 2011.

PIMENTEL-GOMES, F. Curso de estatística experimental. São Paulo: Nobel, 1990. 467 p.

PINAZA, L. A. Cadeia produtiva do milho. Brasília: Ministério da Agricultura, Pecuária e Desenvolvimento. 2007. 108 p.

PIÑA-RODRIGUES, F. C. M.; LOPES, B. M. Potencial alelopático de Mimosa caesalpinaefolia Benth sobre sementes de Tabebuia alba (Cham.) Sandw. Floresta e Ambiente, Seropédica, v. 8, n. 1, p. 1-10, 2001.

RAZAVI, S. M. Plant coumarins as allelophatics agents. International Journal of Biological Chemistry, Pakistan, v, 5, n. 1, p. 86-90, 2011.
REJILA, S.; VIJAYAKUMAR, N. Allelopathic effect of Jatropha curcas on selected intercropping plants (Green Chilli and Sesame). Journal of Phytology, Humnabad, v. 3, n. 5, p. 01-03, 2011.

RIZVI, S. J. N.; RIZVI, V. Allelopathy: basic and applied aspects. London: Chapman \& Hall, 1992. 480 p.

SAIZ, P. Cultura de girassol: produção de biodiesel e outras utilidades. 2009. Disponível em <http://hotsites.sct.embrapa.br/ prosarural/programacao/2009/cultura-do-girassol-2013-producaode-biodiesel-e-outras-utilidades-1>. Acesso em: 23 agosto 2011.

SANTOS, C. C. dos; SOUZA, I. F. de; ALVES, L. W. R. Efeitos de restos culturais de milho sobre o crescimento de plantas de cafeeiro (Coffea arabica L.). Ciência \& Agrotecnologia, Lavras, v. 27, n. 5, p. 991-1001. 2003.

SILVA, H. L.; TREZZI, M. M.; MARCHESE, J. A.; BUZZELLO, G.; MIOTTO, Jr. E.; PATEL, F.; DEBASTIANI, F.; FIORESE, J. Determinação de espécie indicadora e comparação de genótipos de girassol quanto ao potencial alelopático. Planta Daninha, Viçosa, v. 27, n. 4, p. 655-663, 2009.

SOUZA-FILHO, A. P. S.; ALVES, S. M. 2002. Alelopatia: princípios básicos e aspectos gerais. Belém: Embrapa Amazônia Oriental, 2002. 260 p.

SOUZA FILHO, A. P. S.; GUILHON, G. M. S. P.; SANTOS, L. S. Metodologias empregadas em estudos de avaliação da atividade alelopática em condições de laboratório - revisão crítica. Planta Daninha, Viçosa, v. 28, n. 3, p. 689-697, 2010.

TAIZ, L.; ZEIGER, E. Fisiologia vegetal. 4 ed. Porto Alegre: Artmed, 2009. $719 \mathrm{p}$

PINTO, T. T.; FORTES, A. M. T.; BONAMIGO, T.; SILVA, J. da; GOMES, F. M.; PILATTI, D. M. Efeitos alelopáticos do exsudado radicular de Amaranthus cruentus L. sobre sementes de Glycine $\max ($ L.) Merril, Zea mays L. e Bidens pilosa L. INSULA Revista de Botânica, Florianópolis, n. 40, p. 13-24, 2011.

WANG, J. C.; WU, Y.; WANG, Q.; PENG, Y. L.; PAN, K. W.; LUO, P.; WU, N. Allelopathic effects of Jatropha curcas on marigold (Tagetes erecta L.). Allelopathy Journal, New York, v. 24, n. 1, p.123-130, 2009.

ZENG, R. S.; MALLIK, A. U.; LUO, S. M. Allelopathy in sustainable agriculure and forestry.

New York: Editora Springer, 2008. 411 p. 